\title{
Comparison of muscle adaptation toward assistive force between the young and elderly.
}

\author{
Nursalbiah Nasir (nursalbiah@salam.uitm.edu.my) $)^{1,2}$, Keisuke Hayashi (d0831.keisuke@gmail.com)1, \\ Ping Yeap Loh (lohpingyeap@gmail.com) ${ }^{3,4}$, Satoshi Muraki (muraki@design.kyushu-u.ac.jp) ${ }^{3}$ \\ ${ }^{1}$ Graduate School of Design, Kyushu University, Japan \\ ${ }^{2}$ Faculty of Mechanical Engineering, Universiti Teknologi MARA, Malaysia \\ ${ }^{3}$ Faculty of Design, Kyushu University, Japan \\ ${ }^{4}$ Research Fellow of Japan Society for the Promotion of Science, Japan
}

\begin{abstract}
Improving our understanding of muscle response towards assistive force is important not only to ensure the effectiveness of assistive devices but also to avoid injury due to excessive assistance. Therefore, the objective of this study was to compare muscle activities towards assistive force during isometric elbow flexion in young and elderly participants. The study involved right-handed young $(n=9,25.3 \pm 2.4$ years) and elderly $(n=9,68.4 \pm 3.3$ years $)$ male participants and determined the maximum voluntary contraction (MVC) and maximum workload for each participant. The participants performed isometric elbow flexion under two conditions of submaximal workload (20\% and $40 \%)$ and three levels of assistive force $(0 \%, 50 \%$, and $100 \%)$. The electromyographic activity of agonist and antagonist muscles decreased with increased levels of assistive force under both workload conditions in both groups. In addition, a more effective reduction in electromyographic activity was observed during $50 \%$ assistance in both muscles for both groups. In summary, assistive force relieved the exertion in the agonist muscle in both young and elderly groups; however, their effects were influenced by the level of assistive force.
\end{abstract}

Keywords: Assistive force, isometric contraction, elderly, electromyography

\section{Introduction}

A decline in physical capacities in the elderly would impair their mobility and reduce their capability to live independently (Cordozo et al., 2013). In order to maintain a good quality of life and to ensure independent living for the elderly, enhancing motion ability is important. Various types of assistive devices are being developed, which use assistive technology (AT) for the upper and lower limbs. $\mathrm{AT}$ is defined as any equipment or system invented or innovated to increase, maintain, and improve the functional capabilities and mobility of elderly and people with disabilities (Berry and Ignash, 2003). Additionally, AT helps to reduce the burden of supporting mobility in the elderly (Zhuang et al., 1998).

AT has been proven to successfully aid the improvement in motion ability. For instance, Miyawaki et al. (2001) reported $28 \%$ and $35 \%$ deficits in biceps brachii (BB) and triceps brachii (TB) muscle activities, respectively, using the arm balancer assistive device. Bellar et al. (2014) reported that the use of an elastic hamstring assistance device resulted in an $11.6 \%$ reduction in mean electromyographic data of the biceps femoris and a 3.5\% reduction in semitendinosus muscle activities during an isometric knee extension exercise.

Nonetheless, human response to external stimuli suc $\mathrm{h}$ as adaptation towards mechanical assistive force, or electrical vibration stimuli, is dependent upon several $f$ actors, such as human physiology and psychology (St Clair Gibson et al., 2001; Mottram et al., 2006). Ther efore, the effectiveness of assistive force cannot be pre dicted technically based on engineering factors. Further more, differences between the expected output forces a nd the assistive force may vary due to the unique ma nner in which humans respond to stimuli, as well as $\mathrm{i}$ ndividual experiences.

Some studies have investigated age-related differences in muscle activity. For example, Galganski et al. (1993) investigated the effects of aging on motor output of the first dorsal interosseous muscle and found that the elderly could not maintain a constant sub-maximal isometric force with 
their index fingers. Kim et al. (2009) found that lower limb orthosis reduced the activity of the biceps femoris and rectus femoris muscles in 10 elderly participants. In another study, Nasir et al. (2017) investigated adaptation of assistive force while performing isometric elbow flexion in young participants. The study results showed that the decline in muscle activity was enhanced with increase in the assistance of agonist muscles. Nonetheless, a $50 \%$ assistive force at $40 \%$ workload showed a more significant effect towards muscle activity deficit as compared to $100 \%$ assistance. This finding indicated that the effectiveness of assistance was influenced by the combination of assistive force and workload.

However, adaptation to the assistive force and effectiveness of this adaption in elderly participants still remain unclear. We hypothesized that the elderly would show lower muscle activity and lesser adaptation towards assistive force in both agonist and antagonist muscles than those shown by younger participants. Hence, our first objective was to determine agonist (BB) and antagonist (TB) muscle activities towards assistive force in isometric contraction and compare these data for young and elderly participants. Our second objective was to investigate the difference between obtained and theoretical values of muscle activities. To achieve this, a simple exercise involving $90^{\circ}$ elbow isometric flexion with lower submaximal load was performed in our investigation because the adaptability of assistance could be clearly identified by excluding factors, such as joint angle and angular velocity.

\section{Methods}

Right-handed young ( $\mathrm{n}=9,25.3 \pm 2.4$ years of age $)$ and elderly ( $\mathrm{n}=9,68.4 \pm 3.3$ years of age) male participants were recruited in this study. Maximum voluntary contraction (MVC) and maximum workload were identified using electromyography and force sensor output, with the forearm at $90^{\circ}$ angle perpendicular to the upper arm in the supinated position.

Subsequently, the electromyographic data were recorded as the participants performed isometric elbow flexion under two conditions of submaximal workload $(20 \%$ and $40 \%$ of maximal workload) and three levels of assistive force $(0 \%, 50 \%$, and $100 \%$ by theoretical effectiveness).
The participants were requested to maintain the designated posture with target submaximal workload for 33 seconds while the assistive force was applied for 11 to 21 seconds. Next, normalized mean electromyographic (\% MVC) data were recorded.

Finally, a three-way repeated measures analysis of variance ( 2 workload x 3 assistive levels x 2 age groups) was conducted to evaluate the effects on electromyographic data and assistive force. Significant interactions were further investigated by post hoc analysis (Bonferroni-corrected comparison).

\section{Results}

\subsection{Agonist muscle (BB) response towards assistive force}

Significant effects of submaximal workload $\left(\mathrm{F}_{(1,16)}=\right.$ 29.12, $\mathrm{p}<0.001)$ and assistive force level $\left(\mathrm{F}_{(2,32)}=69.39\right.$, $\mathrm{p}<0.001$ ) were found in the agonist muscle (Figure 1). The most effective adaptation was found at $50 \%$ assistive force level. However, the main effect of age groups was not significant.

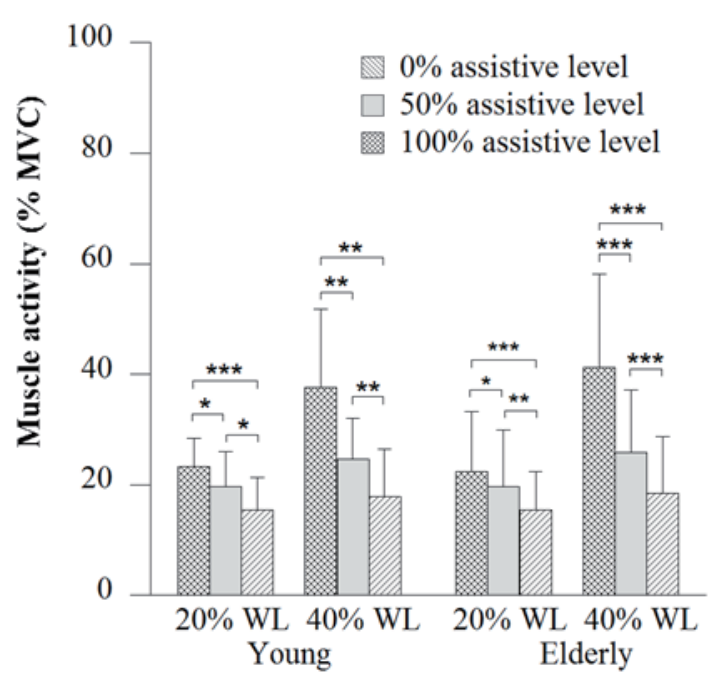

Figure 1. Muscle activity (\% MVC) of agonist muscle in young and elderly participants at different workloads (WL). $\left(* * * \mathrm{p}<0.001,{ }^{* *} \mathrm{p}<0.01,{ }^{*} \mathrm{p}<0.05\right)$. 


\subsection{Antagonist muscle (TB) response towards assistive} force

Significant effect of submaximal workload $\left(\mathrm{F}_{(1,16)}=25\right.$, $\mathrm{p}<0.001)$ and assistive force $\left(\mathrm{F}_{(1.26,20.16)}=10.04, \mathrm{p}<0.01\right)$ were observed in the antagonist muscle (TB). Similar to the agonist muscle (BB), the main effect of age groups was not significant for antagonist muscle. Adaptation towards assistance was found to be most effective at $50 \%$ assistance. However, an increase in \% MVC at $40 \%$ submaximal workload during $100 \%$ assistance was only observed in the elderly group (Figure 2).

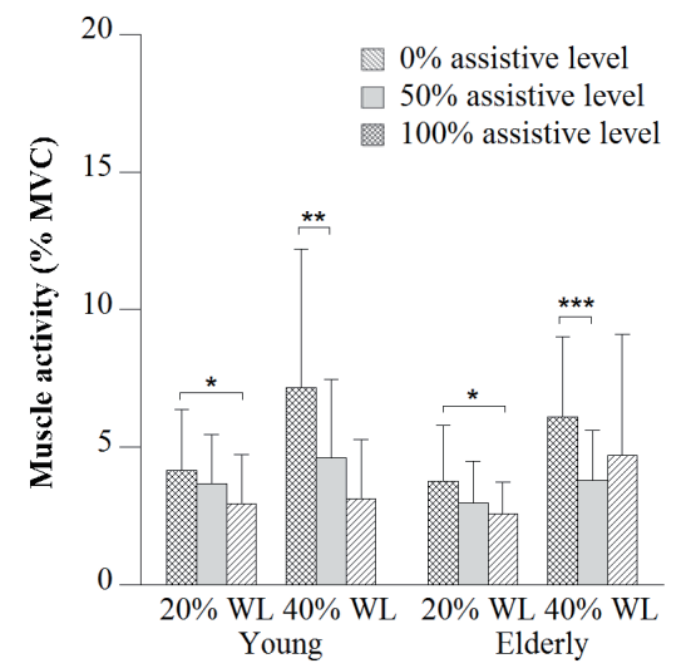

Figure 2. Muscle activity (\% MVC) of antagonist muscle in young and elderly participants at different workloads (WL) $(* * * \mathrm{p}<0.001, * * \mathrm{p}<0.01, * \mathrm{p}<0.05)$.

\section{Discussion}

These results showed that the elderly participants could adapt to assistive force as good as young participants could. Thus, the assistive force relieved the exertion of the agonist muscle irrespective of age. Although the effectiveness of assistive force level was found to be lower than the theoretical value (Figure 1), a $50 \%$ assistive force was found to be the most effective as compared to a $100 \%$ assistive force. This effectiveness of adaptation could be influenced by the co-contraction and torque generation at the elbow joint.

In this study, the assistive force was exerted on the middle part of the forearm. The exertion made participants feel an upward force being transferred from the assistive force to the cable attached to the forearm. Maintaining and stabilizing the $90^{\circ}$ elbow flexion required the participants to resist the upward force by adjusting the forearm, unconsciously moving the arm to correct the position, and activating the agonist and antagonist muscle to create torque at the elbow joint. Both these factors could increase the electromyography signal, thus reducing the assistive force effectiveness.

The antagonist muscle (TB) indicated smaller \% MVC (less than 8\% MVC) than the agonist muscle. However, the elderly group showed a higher \% MVC of antagonist muscle during $40 \%$ workload with $100 \%$ assistive force (Figure 2). The antagonist muscle co-contracts to maintain the required posture and elbow joint stability (Doheny et al., 2008; Rudroff et al., 2010) during isometric contraction, thus showing the muscle activity. In general, the ability to control muscle tension tends to decline with advancing age (Porter, Vandervoot and Lexell, 1995; Yamada et al, 2000; Bazzucchi et al., 2004). Thus, the increase in muscle weakness in the elderly could be a contributing factor to the age related increase in muscle activity.

\section{Acknowledgements}

We would like to express our gratitude to Irma Nur Afiah, Jinghong Xiong, and Wen Liang Yeoh for their assistance in the experiment. This work was supported by the JSPS KAKENHI (Grant Number JP15K14619 and JP17H01454).

\section{References}

Bellar, D., LeBlanc, N. and Judge, L. W., Examination of muscle activity with an elastic hamstring assistance device, Health Research and Reviews, 1(1), 20-24, 2014.

Bazzucchi, I., Felici, F., Macaluso, A. and De Vito, G., Differences between young and older women in maximal force, force fluctuations, and surface emg during isometric knee extension and elbow flexion. Muscle Nerve, 30, 626-635, 2004.

Berry, B.E. and Ignash, S., Assistive technology: providing independence for individuals with disabilities, Rehabilitation Nursing, 28(6), 6-14, 
2003.

Cardozo, A.C., Gonçalves, M., Hallal, C.Z. and Marques, N.R., Age-related neuromuscular adjustments assessed by EMG, Electro diagnosis in New Frontiers of Clinical Research, Associate Prof. Dr. Hande Turker (Ed.), InTech, 2013.

Doheny, E. P., Lowery, M. M., Fitzpatrick, D. P. and O'Malley, M. J., Effect of elbow joint angle on force-EMG relationships in human elbow flexor and extensor muscles. Journal of Electromyography and Kinesiology: Official Journal of the International Society of Electrophysiological Kinesiology, 18(5), 760-770, 2008.

Galganski, M. E., Fuglevand, A. J. and Enoka, R. M., Reduced control of motor output in a human hand muscle of elderly subjects during submaximal contractions, Journal of Neurophysiology, 69(6), 2108-2115, 1993.

Kim, K., Kang, S.R., Heo, M., Kim, N.G. and Kwon, T.K., "Characteristics of the muscle activities for the elderly wearing the lower limb orthosis during gait on the treadmill", Proceedings of ICROS-SICE International Joint Conference, (pp. 519-522), Fukuoka. Kyushu. 2009.

Miyawaki, K., Sato, T., Iwami, T., Obinata, G., Shimada, Y., and Chida, S., Evaluation of utility of the "arm-balancer" arm support system, Journal of Life Support Engineering, 21(1), 3-9, 2009.

Mottram, C. A., Maluf, K. S., Stephenson, J. L., Anderson, M. K., and Enoka, R. M., Prolonged vibration of the biceps brachii tendon reduces time to failure when maintaining arm position with a submaximal load, Journal of Neurophysiology, 95(2), 1185-1193, 2006.

Porter, M. M., Vandervoort, A. A. and Lexell, J., Aging of human muscle: structure, function and adaptability. Scand J Med Sci Sports, 5, 129-142, 1995.

Rudroff, T., Justice, J. N., Holmes, M. R., Matthews, S. D. and Enoka, R. M., Muscle activity and time to task failure differ with load compliance and target force for elbow flexor muscles, Journal of Applied Physiology, 110(1), 125-136, 2011.

St Clair Gibson, A., Lambert, M. L. and Noakes, T. D., Neural control of force output during maximal and submaximal exercise. Sports Medicine (Auckland, N.Z.), 31(9), 637-650, 2001.

Yamada, H., Okada, M., Nemoto, S., Shiozaki, T., Kizuka, T., Kuno, S. and Masuda, T., Effects of aging on EMG variables during fatiguing isometric contractions. J Hum Ergol, 29,7-14, 2000.

Zhuang, Z., Stobbe, T. J., Hsiao, H., Collins, J. W. and Hobbs, G. R., Biomechanical evaluation of assistive devices for transferring residents. Applied Ergonomics, 30(4), 285-294, 1999. 